\title{
ANY FIVE POINTS LIE IN A 3-SPACE
}

Zh.G. Nikoghosyan

Candidate of Science, senior researcher, Institute for Informatics and Automation Problems, National Academy of Sciences,

P. Sevak 1, Yerevan 0014, Armenia

ЛЮБЫЕ ПЯТЬ ТОЧЕК ЛЕЖАТ В З-ПРОСТРАНСТВЕ

Никогосян Жора Гегамович

канд. физ.-мат. наук, старший научный сотрудник, институт проблем информатики и автоматизации, национальная академия наук республики Армении,

П. Севак 1, Ереван 0014, Армения 
Аннотация. В аксиоматических формулировках каждые две точки лежат на (прямой) линии, каждые три точки лежат на плоскости, а каждые четыре точки лежат в трехмерном пространстве (3-пространстве). В этой статье мы показываем, что каждые пять точек также лежат в 3-пространстве, откуда следует, что каждое множество точек также лежит в 3-пространстве. Другими словами, 3-пространство занимает все пространство. Доказательство основано на следующих четырех аксиомах: 1) каждые две различные точки определяют единственную линию, 2) каждые три различные точки, не лежащие на прямой, определяют единственную плоскость, 3 ) если $A$ и $B$ две разные точки в 3 пространстве, то прямая, определенная точками $A$ и $B$, полностью лежит в этом 3 -пространстве, 4$)$ если $F_{1}, F_{2}, F_{3}-$ три различные точки в 3 -пространстве, не лежащие на прямой, то плоскость, определяемая точками $F_{1}, F_{2}, F_{3}$, целиком лежит в этом 3-пространстве.

Abstract. In axiomatic formulations, every two points lie in a (straight) line, every three points lie in a plane and every four points lie in a three-dimensional space (3-space). In this paper we show that every five points lie in a 3-space as well, implying that every set of points lie in a 3-space. In other words, the 3-space occupies the entire space. The proof is based on the following four axioms: 1) every two distinct points define a unique line, 2) every three distinct points, not lying on the line, define a unique plane, 3 ) if $A$ and $B$ are two distinct points in a 3-space, then the line defined by the points $A, B$, entirely lies in this 3 -space, 4 ) if $F_{1}, F_{2}, F_{3}$ are three distinct points in a 3 -space, not lying in a line, then the plane defined by the points $F_{1}, F_{2}, F_{3}$, lies entirely in this 3 -space.

Keywords: point, line, plane, 3-space, non planar graph.

Ключевые слова: точка, прямая, плоскость, 3-пространство, непланарный граф. 


\section{Introduction}

All definitions of the points, lines, planes and their 3-analogs (3-space) are circular in nature since they depend on concepts which themselves must have definitions, a dependence which cannot be continued indefinitely without returning to the starting point. To avoid this vicious circle certain concepts must be taken as primitive concepts; terms which are given no definition. When these concepts are primitive, their behavior and properties are dictated by the axioms which they must satisfy.

The points, lines, planes (induced by two perpendicular lines) and 3-spaces (induced by three mutually perpendicular lines) have certain properties which relate these notions to other points, lines, planes and 3-spaces. For example,

a) for any two distinct points, there is a unique line containing them,

b) for any three distinct points either there are infinite number of planes containing them (when these points lie in a line) or a unique such plane,

c) two distinct planes are either parallel or they intersect in a line,

d) a line is either parallel to a plane, intersects it at a single point, or is contained in the plane.

The 3-space serves as a three-parameter model of the physical universe, that is, the spatial part without considering time.

In axiomatic formulations, every two points lie in a (straight) line, every three points lie in a plane and every four points lie in a three-dimensional space (3-space). 
In this paper we prove the following.

Theorem 1. Every five points lie in a 3-space.

We immediately obtain the following three corollaries.

Corollary 1. Every set of points lie in a 3-space.

Corollary 2. The maximum number of mutually perpendicular lines is three.

Corollary 3. The 3 -space is unique.

In the proof of Theorem 1, we use only the following four statements (axioms).

Axiom 1. Every two distinct points define a unique line.

Axiom 2. Every three distinct points, not lying in a line, define a unique plane.

Axiom 3. If $F_{1}, F_{2}$ are two distinct points in a 3 -space, then the line defined by the points $F_{1}, F_{2}$, lies entirely in this 3 -space.

Axiom 4. If $F_{1}, F_{2}, F_{3}$ are three distinct points in a 3-space, not lying in a line, then the plane defined by the points $F_{1}, F_{2}, F_{3}$, lies entirely in this 3 -space.

We use [1] and [2] for terminology and notation not defined here.

\section{Proof of Theorem 1.}


Let $R^{3}$ be a 3 -space. Assume the theorem is false. Then we can choose a point $A$ in a space with $A \notin R^{3}$. Denote by $W$ the set of all possible points $X$ with $X \notin R^{3}$. Let $D$ be a 3-dimensional object (it can be a ball, a cube, a non-planar graph embedded in $R^{3}$ without edge crossings, and so on) in $R^{3}$ and let $B$ be a point with $B \in D$. The points $A$ and $B$ define a unique straight line m (Axiom 1).

Assume first that m contains another point $B^{\prime}$ of $R^{3}$ other than $B$. By Axiom 3, $m$ lies entirely in $R^{3}$, implying that $A \in R^{3}$, a contradiction. Therefore, the line $m$ intersects the object $D$ and $R^{3}$ only at the unique point $B$. By the same reason,

(1) if $B_{1}$ and $B_{2}$ are two distinct points in $D$, then the lines defined by the couples $\left(A, B_{1}\right)$ and $\left(A, B_{2}\right)$, are distinct as well.

Now let $C_{1}, C_{2}$ and $C_{3}$ be three distinct points in $D$. The couples $\left(A, C_{1}\right),\left(A, C_{2}\right)$ and $\left(A, C_{3}\right)$ define three unique lines $m_{1}$, $m_{2}$ and $m_{3}$, respectively. Further, choose any three points $C_{1}^{\prime}, C_{2}^{\prime}$ and $C_{3}^{\prime}$ on the lines $m_{1}, m_{2}$ and $m_{3}$ such that $C_{i}$ is located between $C_{i}^{\prime}$ and $A$ for each $i \in\{1,2,3\}$. We can assume that $C_{1}^{\prime}, C_{2}^{\prime}$ and $C_{3}^{\prime}$ do not lie in a line and therefore can define some plane $p$ (Axiom 2). Recalling that the line $m$ intersects $D$ and $R^{3}$ at a unique point $C_{i}$ for each $i \in\{1,2,3\}$, we conclude that $C_{1}^{\prime}, C_{2}^{\prime}, C_{3}^{\prime} \in$ $W$ and the plane $p$ lies entirely in $W$ (Axiom 4). The plane $p$ can be considered as a 2-dimensional projection screen (plane) with center $A$, and the points $C_{1}^{\prime}, C_{2}^{\prime}$ and $C_{3}^{\prime}$ can be considered as the images of $C_{1}, C_{2}$ and $C_{3}$ on $p$, respectively. Since $C_{1}, C_{2}$ and $C_{3}$ are distinct, their images $C_{1}^{\prime}, C_{2}^{\prime}$ and $C_{3}^{\prime}$ by (1) are distinct as well. So, on 2-dimensional plane $p$ we have the plane image of 3dimensional object $D$. Then we conclude that some observer, located in $A \in W$, can see the 2-dimensional picture of a 3dimensional object $D$ with its all points. In particular, non-planar graphs $K_{5}$ (the complete graph on 5 vertices) or $K_{3,3}$ (the complete bipartite graph with three vertices in each part) can be seen on a plane $p$ as planar graphs. But it is well-known in graph 
theory that these two graphs cannot be pictured on a plane in such a way that no edges cross each other [1]. This contradiction proves that $A \in R^{3}$ and therefore all the points of the space lie in $R^{3}$. Theorem 1 is proved.

\section{Conclusion.}

Actually, the 3-space occupies all the possible points of the space. The space, where we live (Solar system), having relatively low matter concentration in the universe, is close to the 3-space with only three dimensions induced by three mutually perpendicular axes.

\section{The Used Literature}

[1] J.A. Bondy and U.S.R. Murty, Graph Theory with Applications, Macmillan, London and Elsevier, New York (1976).

[2] R. Hartshorne, Geometry: Euclid and Beyond, New York: Springer-Verlag, 2000. 